\title{
Gravity changes from a stress evolution earthquake simulation of California
}

\author{
T. J. Hayes, ${ }^{1}$ K. F. Tiampo, ${ }^{1}$ J. B. Rundle, ${ }^{2}$ and J. Fernández ${ }^{3}$ \\ Received 7 October 2005; revised 1 May 2006; accepted 12 June 2006; published 29 September 2006.
}

[1] The gravity signal contains information regarding changes in density at all depths and can be used as a proxy for the strain accumulation in fault networks. A stress evolution time-dependent model was used to create simulated slip histories over the San Andreas Fault network in California. Using a linear sum of the gravity signals from each fault segment in the model, via coseismic gravity Green's functions, a time-dependent gravity model was created. The steady state gravity from the long-term plate motion generates a signal over 5 years with magnitudes of $\pm \sim 2 \mu \mathrm{Gal}$; the current limit of portable instrument observations. Moderate to large events generate signal magnitudes in the range of $\sim 10$ to $\sim 80 \mu \mathrm{Gal}$, well within the range of ground-based observations. The complex fault network geometry of California significantly affects the spatial extent of the gravity signal from the three events studied.

Citation: Hayes, T. J., K. F. Tiampo, J. B. Rundle, and J. Fernández (2006), Gravity changes from a stress evolution earthquake simulation of California, J. Geophys. Res., 111, B09408, doi:10.1029/2005JB004092.

\section{Introduction}

[2] Currently, there are numerous fault models which describe the local effects due to topography, stress and strain fields [Chinnery, 1961, 1963; Rundle and Jackson, 1977; Okada, 1985, 1992], and the resultant Coulomb stress field of an earthquake after the event has occurred [King et al., 1994]. Such models have proved useful for a wide variety of applications such as the potential trigger to rupture [Stein et al., 1994]; however, they lack the ability to change over time as they are not models of the seismic evolution for a fault network with complex geometry. Smith and Sandwell [2003, 2004, 2006], have used known slip histories over approximately the last 200 years to calculate how the Coulomb stress field has changed over time using a more efficient form of the semianalytic viscoelastic expressions of Rundle and Jackson [1977]. Such models provide snapshots of the fault system for a given set of input parameters, but they do not dynamically update those parameters in the same manner as a stress evolution model for the system.

[3] Rundle and Kanamori [1987] recognized that the segment, or asperity, model of an earthquake relies on the individual segment's coseismic stress change and its interaction with neighboring segments that determine the segment slip. This was developed into a stress evolution model [Rundle, 1988a, 1989b] whereby a fault network for

\footnotetext{
${ }^{1}$ Department of Earth Sciences, University of Western Ontario, London, Ontario, Canada.

${ }^{2}$ Center for Computational Science and Engineering, University of California, Davis, California, USA.

${ }^{3}$ Institutio de Astronomía y Geodesia (CSIC-UCM), Facultad de CC. Matemáticas, Ciudad Universitaria, Madrid, Spain.

Copyright 2006 by the American Geophysical Union. 0148-0227/06/2005JB004092\$09.00
}

Southern California was allowed to evolve over thousands of years using long-range stress interactions to update the system during each time step. While the above model is not currently a forecast, it does exhibit realistic dynamics observed in the Southern California system; its latest incarnation has 650 segments and includes the northern section of the San Andreas [Rundle et al., 2004]. Ward [2000] developed a time-dependent model for 3000 year cycles of earthquakes in the San Francisco Bay Area, and demonstrated the applicability of such models to hazard analysis.

[4] The connection from a time-dependent model to a forecast requires the assimilation of observational data into the model in order to properly tune the simulation to present/realistic values. The most common types of observations for the strike-slip San Andreas Fault System (SAF) are historical earthquakes from paleoseismic data preserved in the rock record [Brown, 1990]; present day seismic patterns [Tiampo et al., 2002]; surface deformation from GPS measurements (e.g., Southern California Integrated GPS Network (SCIGN)); and interferometric synthetic aperture radar (InSAR) [Massonnet et al., 1993]. The unifying aspect of all these data types is that they are all surface observations: We are left to infer the processes at depth based on the surface expression (e.g., seismicity rates, deformation) of the underlying deterministic dynamical variables at depth (e.g., stress, strain, friction). Rarely is gravity data used for continuous system level observations.

[5] Okubo [1991, 1992] developed the analytic coseismic gravity Green's functions for a fault within an elastic halfspace and further suggested that the Bouguer gravity anomaly be used as a proxy for the accumulation of strain at depth. Unlike strain measurements at the surface, gravity measurements made at the surface contain information from all depths of the underlying medium. Tullis [1996] demon- 
strated that the modeled strain rate for Parkfield, California, at depth is below the current detectability limit of strainmeters at the surface; a result of the hypocentral depth relative to the strainmeter site. Conversely, gravity data can be directly observed and easily collected for system level resolution through various methods: field campaign, permanent station deployment, or possibly periodic airborne surveys. For strike-slip fault networks, values range from $\sim 10$ to $\sim 70 \mu \mathrm{Gal}$; the Scintrex CG-5 Autograv portable gravimeter has standard $1 \mu \mathrm{Gal}$ resolution, and permanent superconducting gravimeters (SGs) have $1 \mathrm{nGal}$ resolution. Moreover, large dip-slip earthquakes, such as the Alaska Good Friday earthquake of 1964, or the Hokkaido 2003 earthquake have been shown to be within the range of satellite observations (e.g., Gravity Recovery And Climate Experiment (GRACE)) [Sun and Okubo, 2004].

[6] Traditionally, gravity studies of earthquakes have focused on the coseismic changes accompanying an earthquake. Recently, Song and Simons [2003] examined the occurrence of large earthquakes in dip-slip systems and their associated free-air gravity anomalies. The study shows remarkable correlation for the occurrence of seismic events and the associated negative free-air gravity anomalies prior to rupture: a consequence of the energy of the system inducing plate flexure above the locked regions most susceptible to unstable slip. These systems typically produce gravity signals an order of magnitude larger than their strike-slip system counterparts, whose preseismic gravity signal is predominantly produced by the resultant dilatation/ compression induced by subsurface stress combined with a relatively small component of vertical surface displacement (compared to dip-slip systems). As such, gravity modeling of strike-slip systems has largely been ignored in the literature for long-term observation purposes.

[7] We propose that the long-term stress loading of a complex fault network, such as the SAF system, in conjunction with the short-term, large magnitude stresses resulting from an earthquake, will produce observable changes in the local gravity signal via the resultant deformation field. Modeling of the gravity signal over large spatial and temporal scales may reveal regions of seismic susceptibility hitherto undetected. Moreover, deviations between the modeled and observed gravity field can highlight regions where previous model assumptions (e.g., friction, medium density) need to be adjusted accordingly. We present here the first attempts at developing a geometrically realistic and time-dependent gravity model for the SAF system.

[8] In presenting our analysis, we will briefly describe the virtual California (VC) stress evolution earthquake simulation of Rundle [1988a, 1988b], followed by an outline of the required gravity Green's functions developed by Okubo [1991, 1992]. We will then present our method of incorporating gravity within the $\mathrm{VC}$ simulation, followed by a discussion of the resulting gravity models for the SAF system: the gravity signal from the steady loading of the SAF; coseismic gravity signals; and a 20 year examination, leading to an event similar to the Brawley, 1940, earthquake.

\section{Evolutionary Earthquake Models}

[9] The development of an earthquake model for prediction and hazard analysis requires a method to update the dynamical input parameters in a physically realistic way, driving the simulation for hundreds to thousands of years [Rundle, 1988a; Ward, 2000]. The need for these evolutionary models is twofold: (1) the deterministic dynamical parameters of the system are inherently unobservable, and (2) the earthquake dynamics are multiscaled with varied spatial and temporal ranges. An evolutionary simulation allows one to investigate the multiscaled dynamics and model the unobservable deterministic parameters in a convenient way.

[10] For earthquake models, the spatial scales range from the grain scale, $1 \mathrm{~nm}$ to $1 \mathrm{~cm}$; the fault zone scale, $1 \mathrm{~cm}$ to $100 \mathrm{~m}$; the fault segment scale, $100 \mathrm{~m}$ to $10 \mathrm{~km}$; the fault system or network scale, $10 \mathrm{~km}$ to $1000 \mathrm{~km}$; and up to the tectonic plate boundary scale, which is in excess of $1000 \mathrm{~km}$. Similarly, we can describe the important temporal scales ranging from the source process time scale, fractions of a second to seconds; to the stress transfer scale, of seconds to years; to the event recurrence timescale, years to thousands of years; up to the fault topology evolution scale, in excess of thousands to millions of years. It becomes evident that, in order to understand earthquake processes over the diverse temporal and spatial ranges, an evolutionary earthquake simulation has many advantages over direct observations alone; in many instances it is the only viable option.

[11] The fundamental and most common observations a time-dependent model requires are the inclusion of current seismic and paleoseismic data for recurrence interval and frictional coefficient estimates; a fault network geometry which reflects the complexity of the system in a realistic manner; as well as rock mechanics properties of the regional earth structure to estimate the aseismic slip factors as well as the bulk properties of the medium used in the simulation. The inclusion of these data sources is essential to any forecasting method attempting to predict events for a specific region. Rundle et al. [2002] refers to this as the data assimilation problem. We note the data collected exists only for a finite number of scales, thus limiting the range of scales available for assimilation into the model. Data which is below the threshold of observational or modeling limits must be treated as a source of noise in the simulation.

\section{Virtual California}

[12] The virtual California (VC) earthquake simulation [Rundle, 1988b; Rundle et al., 2002] is a backslip model whereby the stress accumulation for each segment is generated from the slip deficit (between the loader plate and each fault segment) acquired during each time step as a result of the long-term loading plate velocity. The model's primary purpose is to investigate the long-range interactions of faults within a complex network and the evolutionary dynamics that drive the system. Its output is a slip history reproducing realistic seismicity patterns as observed in the SAF system. As such, the model concentrates on the fault network, or system, spatial scale, while enabling one to investigate earthquakes along the SAF on the event recurrence timescale.

[13] It is presently impossible to know the current state of the stress and strain fields for California with the accuracy required by a deterministic model. Moreover, the deterministic dynamical expressions of an earthquake system are 
often unknown and likely nonlinear. Instead of attempting to solve the (unknown) nonlinear dynamics ab initio, VC employs a cellular automaton (CA) method whereby the nonlinearity of the system is treated via a stochastic algorithm, obviating the need to solve deterministic expressions directly. The VC simulation is modeled such that each fault segment is treated as a cell. The neighborhood of each cell in $\mathrm{VC}$ allows for long-range interactions to take place and is of the order $N$, where $N$ is the number of segments in the model and thus the number of degrees of freedom in the system. The order- $N$ neighborhood implies two key assumptions in the model: each segment interacts with all other segments in the system, and the state of each segment itself is also required to properly update the backslip calculation.

[14] The complex fault network geometry of California is represented by 650 fault segments which have been known to fail in the past [Deng and Sykes, 1997]. Each segment included in the model is approximately $10 \mathrm{~km}$ in length, and has a depth of exactly $15 \mathrm{~km}$. Figure 1 shows the segments which are used in VC for this study. A complete listing of the of the geometry of the fault segments used in $\mathrm{VC}$ for this study is given by Rundle et al. [2004, Table 1].

[15] Much of the observational data for use in $\mathrm{VC}$ is limited to the fault network and recurrence interval timescales and is subsequently averaged where appropriate. This allows more direct assimilation of the observational data into the model. The process of data assimilation for the $\mathrm{VC}$ simulation has been extensively discussed in the works of Rundle et al. [2000, 2002, 2004], and the reader is referred to those articles for a complete discussion.

\subsection{Simulation Algorithm}

[16] Once the data assimilation is complete, we are ready to begin the simulation process. The simulator consists of three main codes, followed by visualization routines. The first two routines generate the input files for the main earthquake simulator. After the simulator has been run for several thousands of years, its output can then be studied via a suite of visualization routines, or subsequently used to create the gravity evolution for the SAF system.

\subsection{Main Simulator Algorithm}

[17] The main earthquake simulator is the CA algorithm and is the transition function [see Weisbuch, 1991] which updates each segment from its state at $t$ to its new state at $t+\delta t$. In general, the transition function contains the rules for how each cell is updated during each step; the transition function can vary from the simple rules outlined in Conway's Game of Life, to the more complex algorithm outlined below.

[18] We start by updating the slip deficit, $\phi(x, t)$, using the relation

$$
\phi(\boldsymbol{x}, t)=s(\boldsymbol{x}, t)-V(\boldsymbol{x}) \delta t
$$

where $s(\boldsymbol{x}, t)$ is slip, $V$ is our long-term slip rate. We then use the previously calculated kinematic stress Green's interaction coefficients, $\mathbf{T}_{i j}^{k l}\left(\boldsymbol{x}-\boldsymbol{x}^{\prime}\right)$, to update the normal stress, $\chi(\boldsymbol{x}, t)$, and shear stress, $\sigma_{x y}(\boldsymbol{x}, t)=\tau_{x y}+\tau_{x y}^{b a c}$, where $\tau_{x y}$ is the component of stress each segment contributes to every other segment due to the new value of $\phi(x, t)$, and $\tau_{x y}^{b a c}$ is a

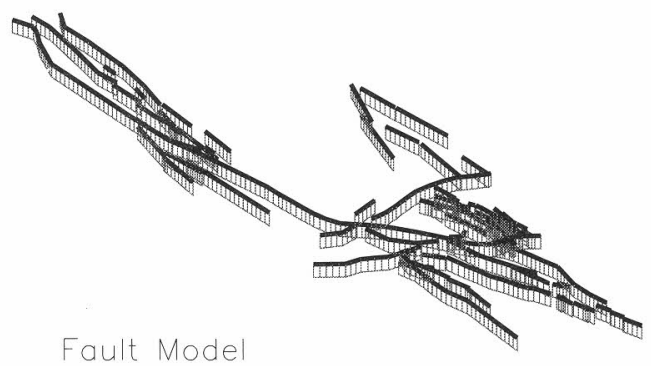

Figure 1. The 650 fault segments display the complexity of the fault network geometry.

background component stress common to the system. We then calculate the Coulomb failure function (CFF) in the typical fashion via

$$
\operatorname{CFF}(\boldsymbol{x}, t)=\sigma_{x y}(\boldsymbol{x}, t)-\mu_{s} \chi(\boldsymbol{x}, t)
$$

[19] At this stage, due to the stress increase from the underlying loading plate there is a fraction of stable, or aseismic, slip which is released, and characterized by the parameter $\alpha$. We use a discretized form of the Coulomb friction law

$$
\frac{\Delta s(\boldsymbol{x}, t)}{\Delta t}=\frac{\Delta \sigma}{K_{L}}\left[\alpha+\delta\left(t-t_{F}\right)\right]
$$

In (3), $\Delta \sigma=\sigma(\boldsymbol{x}, t)-\sigma^{R}(V)$ is the velocity-dependent stress drop associated with the aseismic slip, and $V=V(\boldsymbol{x}) . K_{L}$ is the stress drop stiffness for each fault segment which is inversely proportional to each segment's average slip rate. $K_{L}$ represents the loader plate spring constant in the model. We further note that $t_{F}$ is any time when the condition $\sigma\left(\boldsymbol{x}, t_{F}\right)=\sigma^{F}(V)$ is met, i.e., the value of stress when failure occurs. The variables, $\sigma^{R}(V)$ and $\sigma^{F}(V)$ can be further parameterized by the coefficients of friction, i.e., $\sigma^{R}(V)=\mu_{k} \chi(\boldsymbol{x}, t)$ and $\sigma^{F}(V)=\mu_{s} \chi(\boldsymbol{x}, t)$. Having updated the system with stable slip, we once again update the stress for the entire system via $\mathbf{T}_{i j}^{k l}\left(\boldsymbol{x}-\boldsymbol{x}^{\prime}\right)$ and calculate $\mathrm{CFF}(\boldsymbol{x}, t)$ for each segment using (2).

[20] The simulation is run as described above, incrementing the stress on the system due to the loader plate velocity until failure occurs on a segment(s). Failure occurs when the condition $\operatorname{CFF}(\boldsymbol{x}, t) \geqslant 0$ is met.

[21] Once the CFF criterion for failure has been met on one segment, we then enter into the failure loop which consists of a series of parallel Monte Carlo sweeps (MCS). During the first MCS we allow unstable slip of magnitude,

$$
\Delta s(\boldsymbol{x}, t)=\frac{\sigma(\boldsymbol{x}, t)-\mu_{k} \chi(\boldsymbol{x}, t)}{K_{T}}(1+\rho)
$$

for each site which meets the failure criterion. The value $\rho$ is a randomly generated variable whose probability density function is uniformly distributed over the interval $( \pm 0.1)$. This allows for up to $10 \%$ of random overshoot/undershoot of slip on the fault. Note here we now use the total spring 
constant, $K_{T}=K_{L}+K_{P}$, where $K_{P}$ is interpreted as the spring constant for each segment with all other segments in the model. Physically, this is done to allow the failing sites to transfer stress to all of the segments in its neighborhood, i.e., the entire fault network. The stress is once again transferred via $\mathbf{T}_{i j}^{k l}\left(\boldsymbol{x}-\boldsymbol{x}^{\prime}\right)$ using the same procedure as before to update the stresses from the backslip calculation. This ends the first MCS.

[22] We must now determine whether or not any other segments are now at failure $\operatorname{CFF}(x, t) \geqslant 0)$ due to the stress acquired from the unstable slip in the first MCS. Slip and stress are once again updated as above and repeated until the end of the MCS procedure, when no new sites are at failure. Healing on a segment is only allowed once all of the slips have been updated. At this point we exit the failure algorithm and return to the main routine. Time is updated from $t$ to $t+\delta t$ and we repeat the entire process until a maximum time, $t=t_{M}$ (defined by the user), is reached. For typical runs of the simulation we set $t_{M}=5000 \mathrm{yr}$. The above procedure was used for all runs used in this study.

[23] Using the virtual California earthquake simulator, we are able to create realistic earthquake evolutions for California over thousands of years. From the slip histories created, one can then model how other aspects of the system evolve. Previous studies that use known slip histories of the SAF system have focused on generating maps of the timedependent Coulomb stress field [Deng and Sykes, 1997; Smith and Sandwell, 2003], or surface deformations that are readily captured by the densely deployed GPS stations throughout California (e.g., SCIGN). This study will develop a model to show the spatial evolution of the gravity signal for California using the simulated earthquake histories generated by VC.

\section{Gravity Green's Functions}

[24] In practice, the true deterministic state variables (e.g., $\sigma(\boldsymbol{x}, t))$ which drive the system dynamics are unobservable at the surface. What is recorded are the surface dynamics resulting from the evolution of the underlying state variables [Rundle et al., 2001]. One variable whose surface expression is a record of the processes at all depths is gravity. Accordingly, Okubo [1992] suggests that one can use the Bouguer gravity anomaly as a proxy for strain accumulation at depth.

[25] By incorporating the Green's functions for gravity changes into $\mathrm{VC}$, we acquire the ability to model the evolution of the gravity changes over extended timescales. The expectation is that the accumulation of the long-period gravity signals may be observable through field mapping excursions. Sun and Okubo [2004] have shown that large subduction zone earthquakes, Alaska (1964, 2002) and Hokkaido (2003), are within the expected observable limits of the Gravity Recovery and Climate Experiment (GRACE) satellites. These large earthquakes are associated with short period signals with a temporal range of $100 \mathrm{~s}$ to $10000 \mathrm{~s}$, much smaller than the evolution of the slow strain build up of $100 \mathrm{yr}$ to $10000 \mathrm{yr}$. The long-term loading plate over time that drives the system will also contribute a significant component over long integration times. We will demonstrate that the gravity signals generated by a strike-slip system, such as the SAF system, are within the range of current portable and permanent field instruments.

[26] The Green's function solutions of Okubo [1991, 1992] allow one to calculate the total gravity change, free-air gravity change, or the dilatational changes for a rectangular fault within a half-space with arbitrary dip. Since the fault segments used in VC are strike-slip rectangular fault planes within an elastic half-space, as described in section 3 , the inclusion of gravity changes within $\mathrm{VC}$ is a natural extension of the simulator's applicability. This section outlines those required expressions of $O k u b o$ [1991, 1992] by which gravity within an elastic half-space is calculated for our model.

\subsection{Point Source Potential Changes}

[27] The analytical solutions of strain and deformation in a half-space were developed by Steketee [1958a, 1958b]; Press [1965], then further extended by Chinnery [1961, 1963], Maruyama [1964], and Mansinha and Smylie [1971] to include different fault types (e.g., strike-slip, dip-slip). These were finally unified into a single notation and coordinate system by Okada [1985, 1992]. In order to find the coseismic gravity Green's functions, Okubo [1991, 1992] calculates the potential changes due to a deformation field and then takes the vertical derivative of those solutions to obtain the gravity values.

[28] The first component Okubo [1992] describes is the change in the potential which occurs solely because of density perturbations in the underlying medium. Physically this can produce dilatational or compressional regimes (i.e., the Bouguer anomaly), and they are described by $-\rho\left[\nabla \cdot \boldsymbol{u}\left(\boldsymbol{r}^{\prime} ; \xi_{3}\right)\right]$. We will refer to this type of potential change as the dilatational component of the total potential field. The second change in the potential arises from the mass displaced vertically $\Delta h$ from the source. This is and denoted as $\Delta \phi$. We will refer to this component as the free-air component of the total gravity. There can also be a component of potential changes associated with the generation of a cavity filled with new material of density $\rho^{\prime}$; this is the cavitation component of the potential and will not be investigated in this study.

\subsection{Gravity Change for a Finite Fault}

[29] To find the solution for a finite fault plane, simplify by finding the changes observable at the surface with a source located at $\xi \equiv \xi_{3}$. Then the slip vector, $\Delta s=\left(U_{1}, U_{2} \cos \delta-U_{3}\right.$ $\left.\sin \delta,-U_{2} \sin \delta-U_{3} \cos \delta\right)$, is defined for a rectangular fault with a length $L$, width $W$, dip angle $0<\delta \leqslant \pi / 2$, and depth $d$. Figure 2 shows the relationship among these variables. Positive values of $U_{1}, U_{2}$, and $U_{3}$ correspond to left-lateral slip, thrusting slip, and tensile motion, respectively; $\delta=\pi / 2$ corresponds to a purely strike-slip fault.

[30] Using the coordinate system in Figure 2, Okubo [1992] obtains for the coseismic change in gravity, $\Delta \boldsymbol{g}$, of a finite fault plane evaluated at the surface, i.e., $x_{3}=0$, a solution of the form

$$
\begin{aligned}
\Delta g\left(x_{1}, x_{2}\right)= & \left\{\rho G\left[U_{1} S_{g}(\xi, \eta)+U_{2} D_{g}(\xi, \eta)+U_{3} T_{g}(\xi, \eta)\right]\right. \\
& \left.+\Delta \rho G U_{3} C_{g}(\xi, \eta)\right\} \|-\beta \Delta h\left(x_{1}, x_{2}\right)
\end{aligned}
$$

where we have used the double vertical notation of Chinnery [1961]. The free-air gravity gradient is given by 


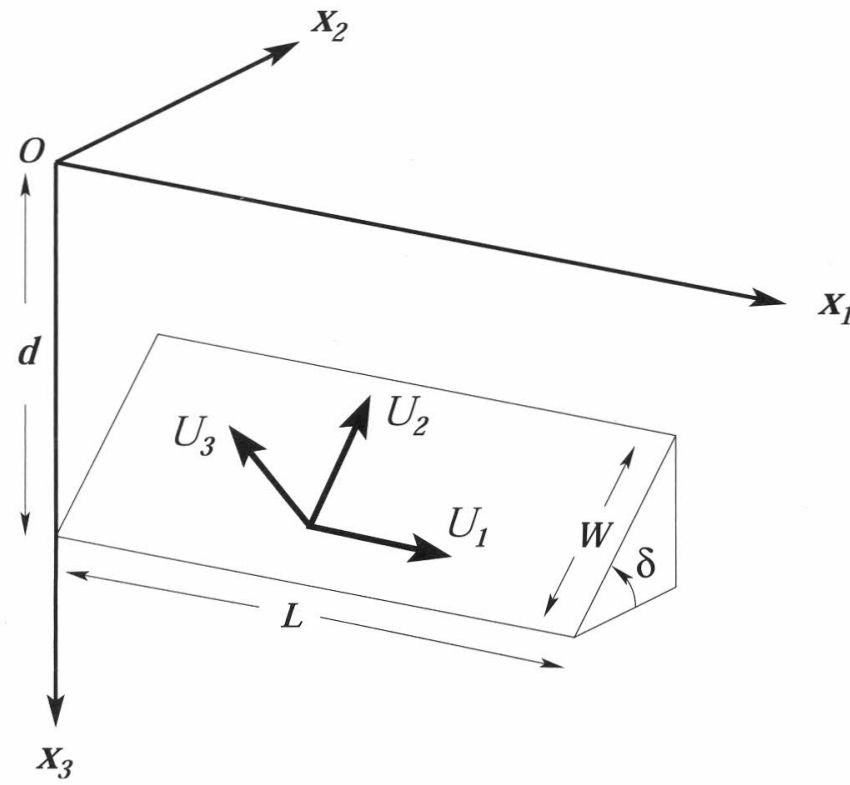

Figure 2. Coordinate system with origin $O$, and the source model geometry used for the calculation of the gravity Green's functions. See text for symbol meanings. Modified after Okubo [1992].

$\beta=0.309 \times 10^{-5} \mathrm{~m} \mathrm{~s}^{-2}, \Delta \rho$ is the difference in density between the cavity filling matter and the original medium, i.e., $\Delta \rho=\rho^{\prime}-\rho$. The functions, $S_{g}(\xi, \eta), D_{g}(\xi, \eta), C_{g}(\xi, \eta)$, and and $T_{g}(\xi, \eta)$ are gravity Green's function contributions from the strike-slip, dip-slip, cavity filling, and tensile components, respectively. For our study, we only require the Green's function for the strike-slip case which is given by

$$
S_{g}(\xi, \eta)=\frac{q \sin \delta}{R}+\frac{q^{2} \cos \delta}{R(R+\eta)}
$$

and the function $\Delta h(\boldsymbol{x})$ in (5), evaluated at $\boldsymbol{x}=\left(x_{1}, x_{2}\right)$, records the elevation change and is given by Okada [1985] as

$$
\begin{gathered}
\Delta h(x)=\frac{1}{2 \pi}\left[U_{1} S_{h}(\xi, \eta)\right] \| \\
S_{h}(\xi, \eta)=\frac{\bar{d} q}{R(R+\eta)}+\frac{q \sin \delta}{R+\eta}-I_{4} \sin \delta
\end{gathered}
$$

where,

$$
\begin{aligned}
& R=\sqrt{\xi^{2}+\eta^{2}+q^{2}} \\
& q=x_{2} \sin \delta-\left(d-x_{3}\right) \cos \delta \\
& \bar{d}=\eta \sin \delta-q \cos \delta \\
& \bar{y}=\eta \cos \delta+q \sin \delta
\end{aligned}
$$

and finally,

$$
I_{4}(\xi, \eta)=(1-2 \nu)[\log (R+\bar{d})-\sin \delta \log (R+\eta)] \sec \delta
$$

where $\nu$ is the Poisson ratio and is set to 0.25 in this study.
[31] For the gravity change free from the effect of the vertical displacement of the ground, $\Delta h\left(x_{1}, x_{2}\right)$, Okubo [1992] provides the following expression:

$$
\begin{aligned}
\Delta g^{*}\left(x_{1}, x_{2}\right)= & \left\{\rho G\left[U_{1} S_{g}^{*}(\xi, \eta)+U_{2} D_{g}^{*}(\xi, \eta)+U_{3} T_{g}^{*}(\xi, \eta)\right]\right. \\
& \left.+\Delta \rho G U_{3} C_{g}(\xi, \eta)\right\} \|
\end{aligned}
$$

where once again, we require only the strike-slip component, namely,

$$
S_{g}^{*}(\xi, \eta)=I_{4} \sin \delta
$$

There are some instances in formulae (5) to (10) where singularities occur. Refer to Okubo [1991, 1992] for instructions pertaining to the treatment of the cases when $\cos \delta=0, \xi=0, q=0$, or $R+\eta=0$; as well as a complete description of the derivations. The outline shown here presents only those aspects of the calculation of the gravity Green's function solutions pertinent to our problem.

\section{Virtual Gravity}

[32] The solutions given by (5) to (10) have proved useful in a wide variety of applications. These expressions assume a single fault plane whose geometry does not change over long distances; a reasonable approximation over regions of several tens of kilometers. However, the assumption ceases to be realistic at the fault network scale where asperities in the medium cause the fault geometry to deviate and develop splays and subfaults as observed in the SAF system. We can obviate this inherent limitation by breaking up the faults in the SC fault network into the "segments" used in the VC simulation and then use a linear sum of their solutions for each segment. Doing so allows us to maintain confidence in the validity of using (5) to (10) over an extended region with multiple faults that exhibit complex geometries. This section outlines the method by which this was achieved now that the necessary components of the theory have been established.

\subsection{Overview of VC's Gravity Calculation}

[33] In calculating the changes in the gravitational field we wish to adhere to several criteria. The first is the principle of least interference we apply by developing a method which imposes the least amount of modification to the main earthquake simulator. Moreover, it removes any subjectivity calculating the gravity from multiple faults over a large network. An ancillary goal was to develop a modular algorithm in order to allow subsequent modification for future endeavors with ease. We applied all these criteria in the following way.

\subsection{Gravity Green's Functions Routine}

[34] The calculation of the gravity changes from the slip histories generated by VC is composed of two components: the steady state (or long-term) signal generated by the continual accumulation of backslip from the underlying loading plate with magnitude, $V(\boldsymbol{x}) \delta t$; and the sudden release of strain energy during unstable slip on the fault (i.e., $\Delta s(\boldsymbol{x}, t))$ as described by (4) in section 3. Accordingly, the first step was to have the virtual gravity routine $(\mathrm{VG})$ 
extract: the slip histories generated by VC; the fault network geometry; and the average fault segment velocities as estimated in the data assimilation process. Once these data were assimilated an observation grid of 10000 points was created to cover all 650 fault segments in the model.

\subsubsection{Calculation of the Steady State Gravity}

[35] The first step in calculating the contribution to the overall gravity from the long-term, steady state component is to artificially create two extensions on the fault segment network at the northernmost and southernmost segments in the network. This is analogous to finite difference problems where the solution cell on the boundary is solved by the use of ghost points, which lie outside the numerical grid, in order to properly calculate the dynamics on the boundaries. Similarly, the introduction of our "ghost segments" acts to mitigate anomalous effects at the extreme boundaries on the fault network. The spatial extent of the northern and southern extensional segments are proportional to a fraction of the average loader plate velocity, $|V|$. More specifically, in the $x_{1}$ direction, spatial extent is proportional to,

$$
\frac{V_{x_{1}}}{|V|}
$$

and the spatial extent in the $x_{2}$ direction is proportional to,

$$
\frac{V_{x_{2}}}{|V|}
$$

[36] Once the segment geometry and observational grid is set, we then calculate the gravity value which would be observed at each of the 10000 grid points on the surface due to the steady state slip from each segment. We note that in this study we can make the following simplifications: all slip motion is strike slip, so that $\Delta \boldsymbol{s}(\boldsymbol{x}, t)=\left(U_{1}, 0,0\right), \delta=\pi / 2$, and there is no cavity filling matter, $\Delta \rho=0$. Applying these changes to (5) and (9) simplifies the expressions significantly yielding

$$
\Delta g\left(x_{1}, x_{2}\right)_{f}=\left\{\rho G\left[U_{1} S_{g}(\xi, \eta)\right]\right\} \|-\beta \Delta h\left(x_{1}, x_{2}\right)
$$

and,

$$
\Delta g^{*}\left(x_{1}, x_{2}\right)_{f}=\left\{\rho G\left[U_{1} S_{g}^{*}(\xi, \eta)\right]\right\} \|
$$

respectively, where the subscript, $f$, indicates that this is the result for the contribution of steady state gravity from a single segment to an observation point located at $\boldsymbol{r}=\left(x_{1}, x_{2}, 0\right)$. The complete solution for the entire network is the linear sum of these contributions from each segment. Therefore we obtain

$$
\Delta \boldsymbol{g}_{\Sigma_{S S}}(\boldsymbol{r})=\sum_{f=1}^{N} \Delta g\left(x_{1}, x_{2}\right)_{f}
$$

and,

$$
\Delta \boldsymbol{g}_{\Sigma_{S S}}^{*}(\boldsymbol{r})=\sum_{f=1}^{N} \Delta g^{*}\left(x_{1}, x_{2}\right)_{f}
$$

for the total gravity and dilatational gravity fields $N$ is the number of segments in the network, and the subscript $\Sigma_{S S}$ indicates the summed steady state solution for the fault network, respectively.

[37] Now that we have developed the expressions for the entire network, we are able to find the gravity from the steady state slip in California. To do so we must calculate $U_{1}$ for (11) and (12); the strike-slip component of the slip vector $\Delta s(\boldsymbol{x})$. As this value of slip is constant over all time steps, we use

$$
U_{1}^{S S}=\Delta s(\boldsymbol{x})_{f}=V(\boldsymbol{x})_{f} \delta t
$$

to find the gravity contribution of steady state slip per unit time step for each fault segment. We note that in the implementation of the algorithm that the output from this

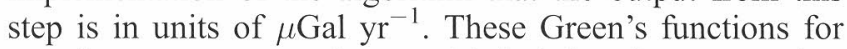
a unit response are then multiplied by the appropriate amount of time under investigation to yield a change in $\mu \mathrm{Gal}$.

\subsubsection{Elastic Contribution to Gravity}

[38] The second contribution, from the purely elastic unstable slip, follows the same method as above with the following exceptions. This component calculates the gravity from unstable slip from each segment in the network, thus the use of artificial ghost segments is unnecessary. To be complete in our analysis, we again use (11) and (12) but now specify that this component is separate from the steady state contribution by using the notation

$$
\Delta \boldsymbol{g}_{\Sigma_{E S}}(\boldsymbol{r})=\sum_{f=1}^{N} \Delta g\left(x_{1}, x_{2}\right)_{f}
$$

and,

$$
\Delta \boldsymbol{g}_{\Sigma_{E S}}^{*}(\boldsymbol{r})=\sum_{f=1}^{N} \Delta g^{*}\left(x_{1}, x_{2}\right)_{f}
$$

for the total gravity and dilatational gravity fields, respectively. The subscript $\Sigma_{E S}$ indicates the summed elastic slip contribution for the fault network.

[39] The main difference from the steady state component is that we are now relying on the time-dependent variable slip $\Delta s(\boldsymbol{x}, t)$ as given by (4). In other words, we set the strike-slip component of the slip vector in (11) and (12) to

$$
U_{1}^{E S}=\Delta s(\boldsymbol{x}, t)_{f}=\left[\frac{\sigma(\boldsymbol{x}, t)-\mu_{k} \chi(\boldsymbol{x}, t)}{K_{T}}(1+\rho)\right]_{f}
$$

for the elastic slip case. In the implementation of this component of the gravity signal, we first calculate the response for unit unstable slip, yielding Green's functions with the units of $\mu \mathrm{Gal} \mathrm{m}{ }^{-1}$. The Green's functions are then multiplied by the modeled slip output by VC to obtain a final result with units of $\mu \mathrm{Gal}$.

\subsubsection{Resultant Gravity Field Changes}

[40] We now have all the necessary components to find the modeled gravity changes over the SAF system using the $\mathrm{VC}$ stress evolution earthquake simulation. All that remains 


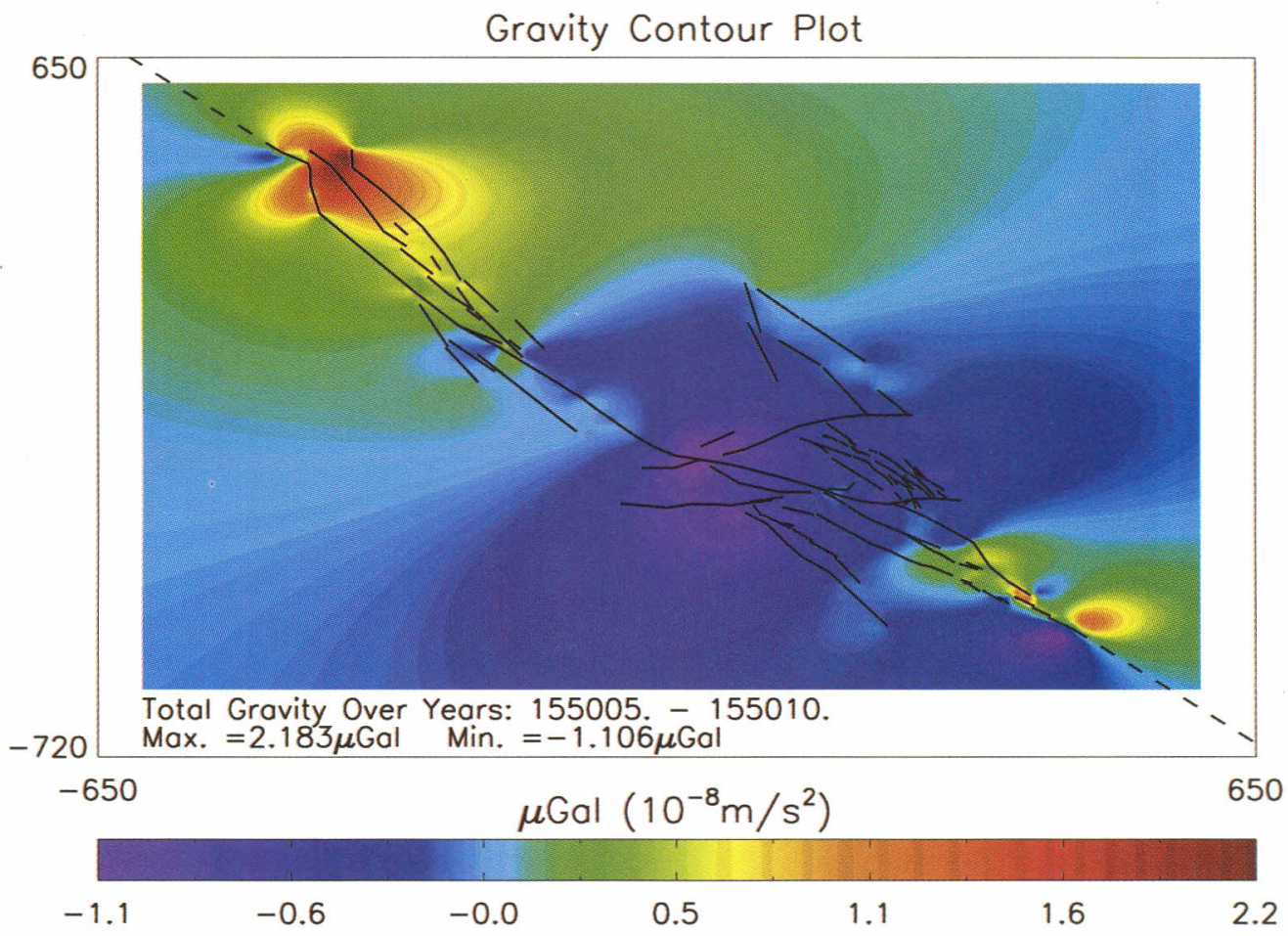

Figure 3. Gravity plot of the steady state field showing the total gravity. The black lines are the 650 fault segments used in this study.

is to sum the steady state and elastic slip components of gravity, yielding

$$
\Delta \boldsymbol{g}_{\Sigma_{T O T}}(\boldsymbol{r})=\left[\Delta \boldsymbol{g}_{\Sigma_{E S}}(\boldsymbol{r})+\Delta \boldsymbol{g}_{\Sigma_{S S}}(\boldsymbol{r})\right]
$$

for the total gravity changes from the steady state and elastic component. We also have,

$$
\Delta \boldsymbol{g}_{\Sigma_{D S L}}^{*}(\boldsymbol{r})=\left[\Delta \boldsymbol{g}_{\Sigma_{E S}}^{*}(\boldsymbol{r})+\Delta \boldsymbol{g}_{\Sigma_{S S}}^{*}(\boldsymbol{r})\right]
$$

for the total dilatational component. To obtain the only freeair gravity contribution to the signal, i.e., the component of gravity due only to the changes in height, $\Delta h$, we subtract (20) from (19) as follows:

$$
\Delta \boldsymbol{g}_{\Sigma_{F A G}}(\boldsymbol{r})=\Delta \boldsymbol{g}_{\Sigma_{T O T}}(\boldsymbol{r})-\Delta \boldsymbol{g}_{\Sigma_{D L L}}^{*}(\boldsymbol{r})
$$

We iterate the calculations (19), (20), and (21), for each of the 10000 observation points, and then we can examine the gravity changes on the fault network.

\section{Results}

\subsection{Steady State Gravity Signal}

[41] Figures 3, 4, and 5 models how the gravity accumulates from only the steady state loading over a 5 year interval. The complex geometry of the fault network is interpreted to be the main mechanism by which the spatial extent of the gravity is affected. The background plate velocity of each segment drives the magnitude of the signal.

[42] The total gravity changes range from 2.18 to $-1.11 \mu \mathrm{Gal}$ for the 5 year period, and the dilatational component of the gravity signal has a range of $1.39 \mu \mathrm{Gal}$ to $-2.92 \mu \mathrm{Gal}$. The free-air gravity values contain the largest magnitude signal, with a range of 5.01 to $-2.48 \mu \mathrm{Gal}$. These values are at the current limits of portable instruments, and within the range of long-term permanent recording devices. Over a 10 year period the spatial extent of the steady state component (not shown) is remarkably similar to the 5 year case. The main difference is that the magnitude of the signal is approximately double. This result was expected as the stochastic unstable slips in the system provide the largest variability of the gravity signal in the elastic halfspace used here.

\subsection{Large Event Gravity Signals}

[43] We now examine the gravity signals generated for large slip events such as the 1906 San Francisco earthquake and the 1857 Fort Tejon earthquake. Figure 6 shows an event similar to the San Francisco earthquake of 1906. It is important to remember that the model's only input are the fault geometry and the assimilated coarse grained variables. The event in Figure 6 arises solely from the evolutionary dynamics and the long-range stress interactions in the simulation and is not prescribed to fail in the manner shown.

[44] The most interesting spatial signal in Figure 6 emerges from the dilatational component of gravity. Here we observe a small, but nonnegligible effect of the steady state signal imposing a slight trend. Moreover, the effect of having the slip occur on multiple segments (thick black lines) as well as the complex geometry affects the shape of the signal. For a perfectly straight fault, one would expect to see an antisymmetric "butterfly" distribution of gravity [see Okubo, 1992, Figure 4b]. The effect of slip in the ConcordGreen Valley area has both mitigated and amplified the 


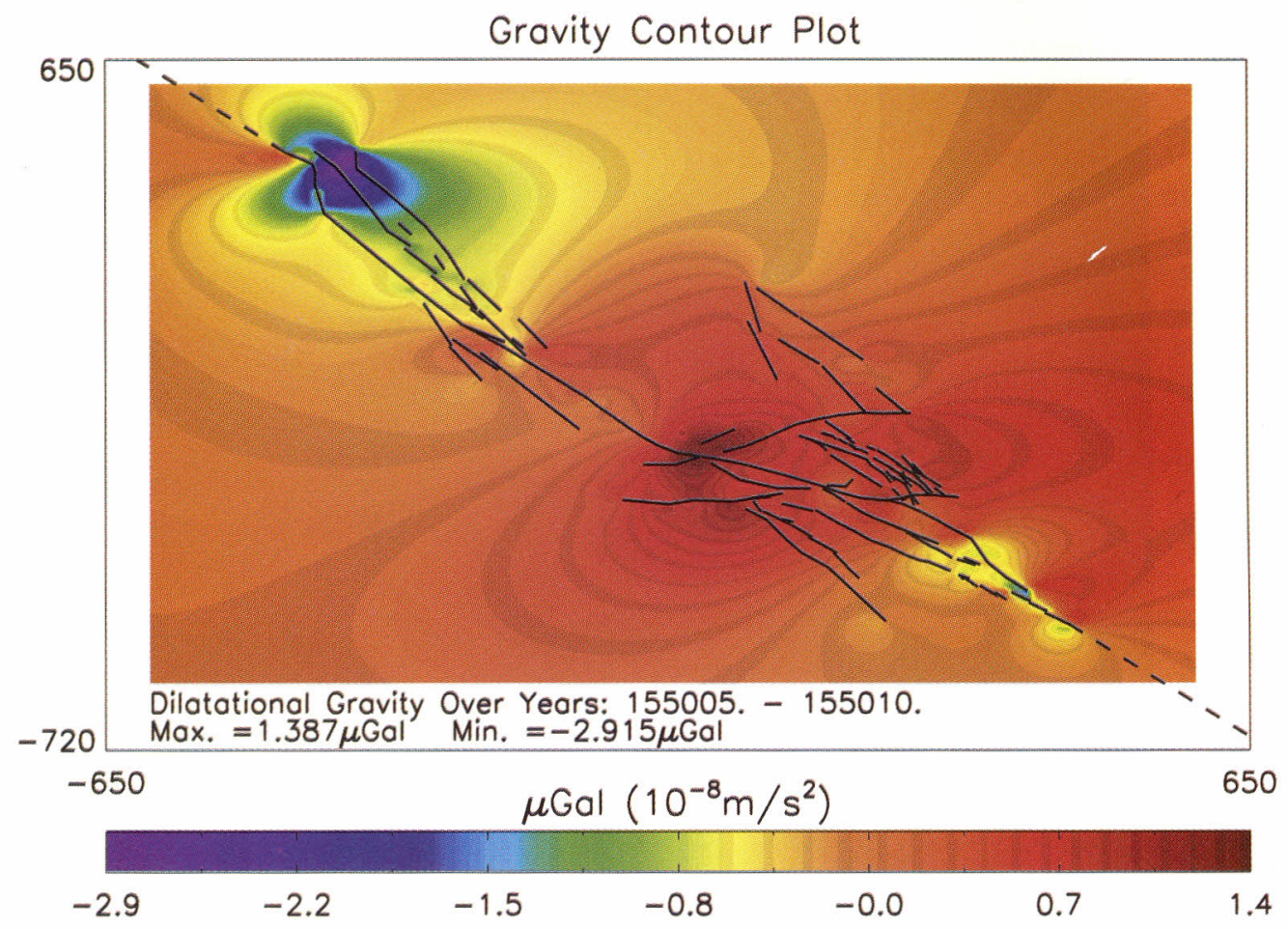

Figure 4. Gravity plot of the steady state field showing the dilatational gravity.

gravity signal in unpredicted ways northeast of the region. The dilatational gravity signal from this event ranges from 43.92 to $-49.08 \mu \mathrm{Gal}$, and the free-air component has similar magnitudes. The elevation changes recorded by the free-air component play a significant role in the gravity signal close to the rupture and are in the opposite sense to the dilatational signal.

[45] For another large event from the simulation, similar to the 1857 Fort Tejon event, we observe even more significant contributions to the gravity signal shape from the fault network geometry. In this case we have very few

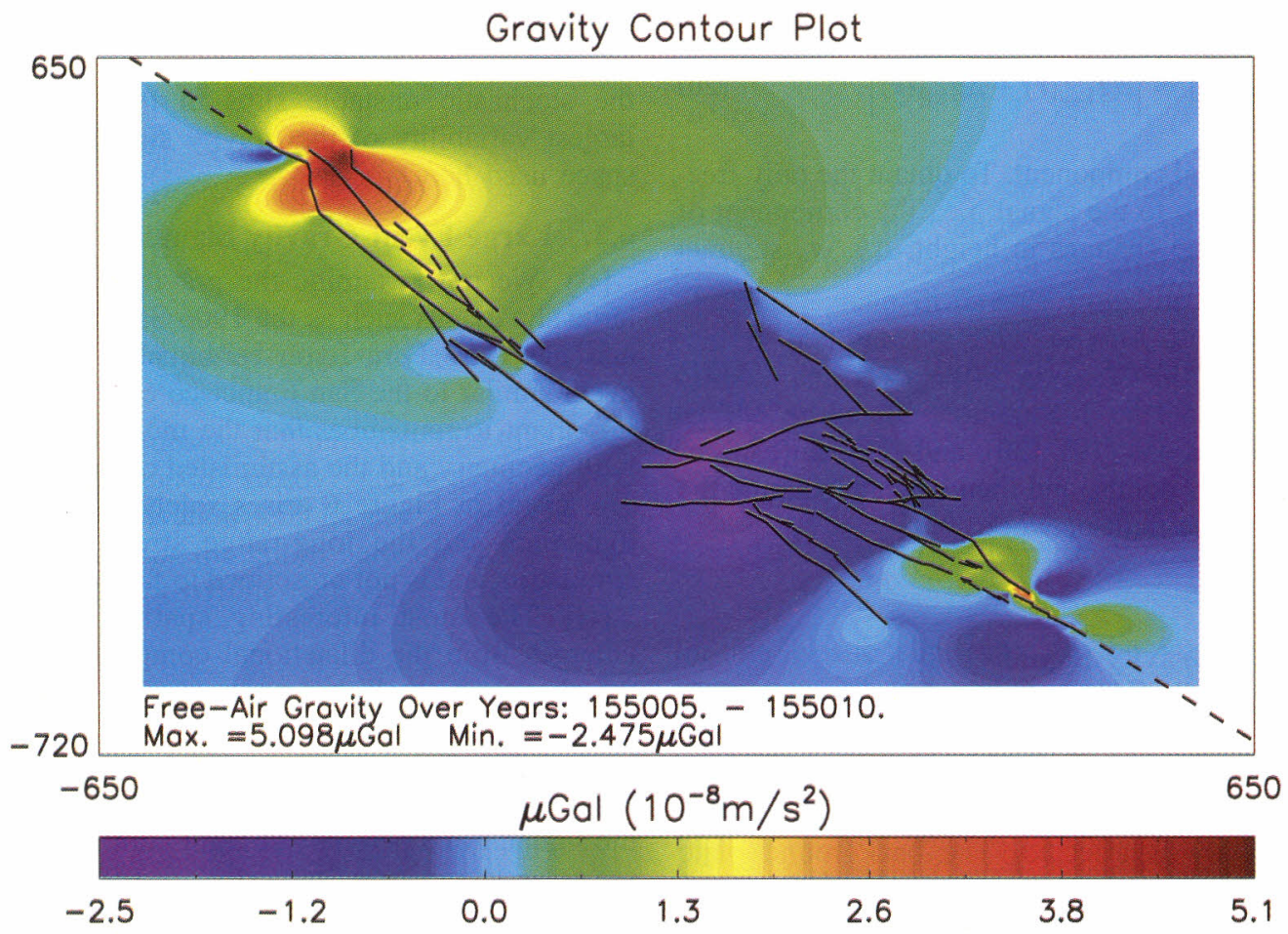

Figure 5. Gravity plot of the steady state field showing the free-air gravity. 

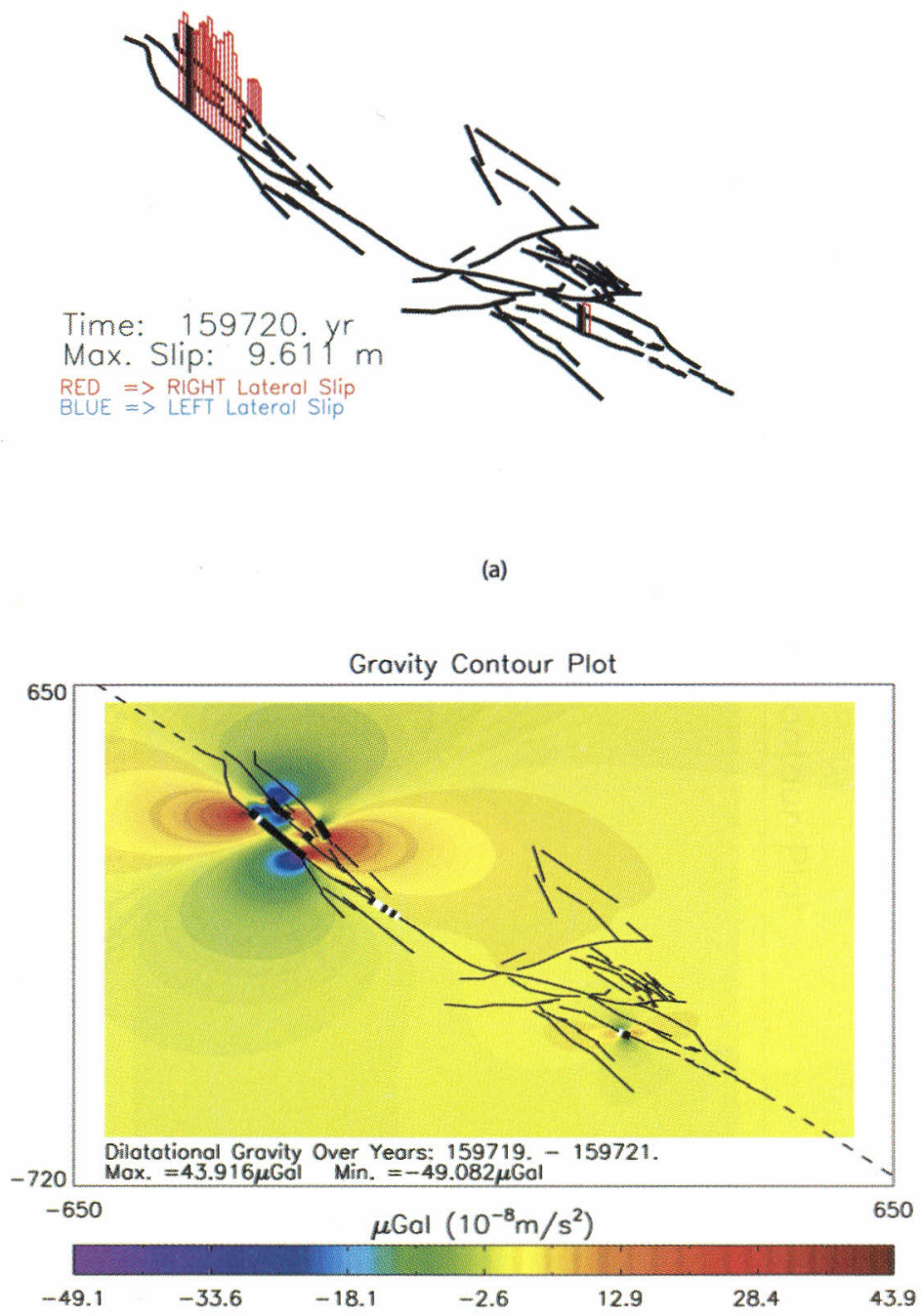

Grovity Contour Plot

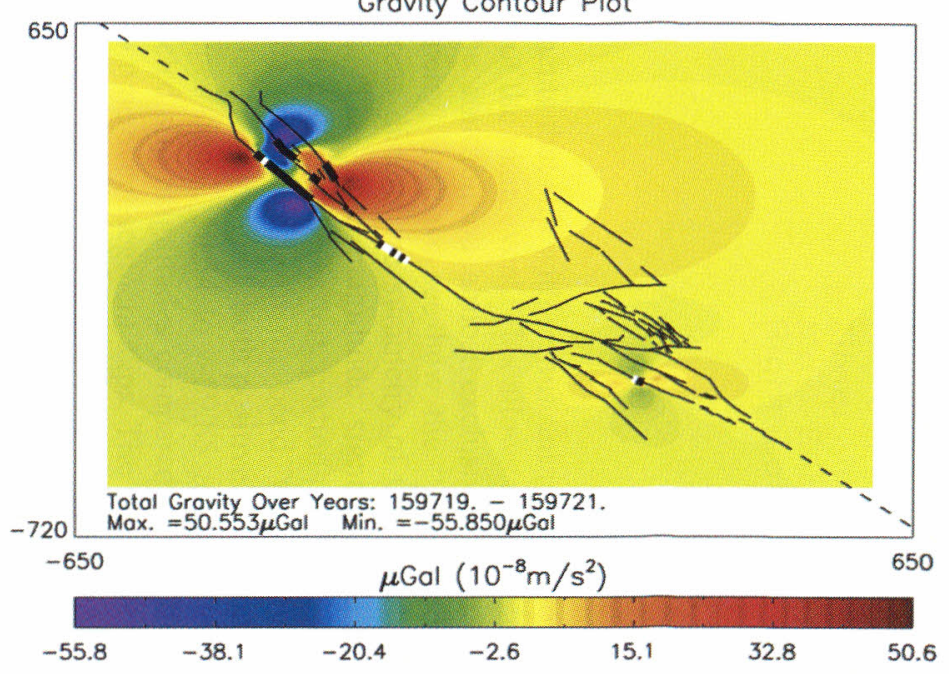

(b)

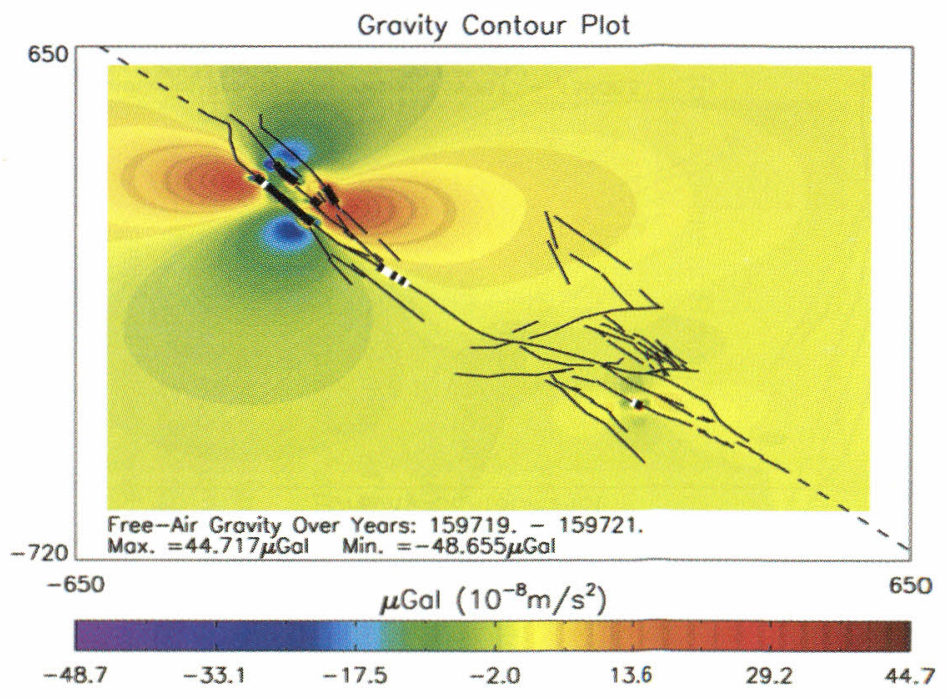

Figure 6. Gravity plots of a large event similar to the San Francisco earthquake of 1906. (a) Total elastic slip from each segment that slipped in the simulation. The height of the bars is proportional to the amount of slip for each segment, and the black bar(s) indicate an initiator site, i.e., hypocenter. (b) to (d) Total gravity signal, the dilatational component, and the freeair components, respectively. The thick black lines indicate segments that slipped during the interval modeled; the thick white lines indicate which segments were initiators, i.e., segments which failed during the first MCS. 


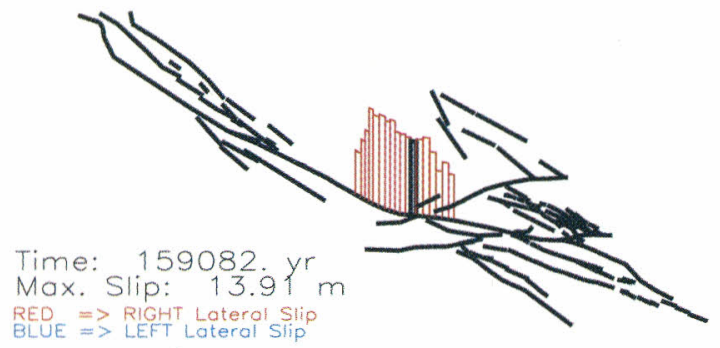

(a)

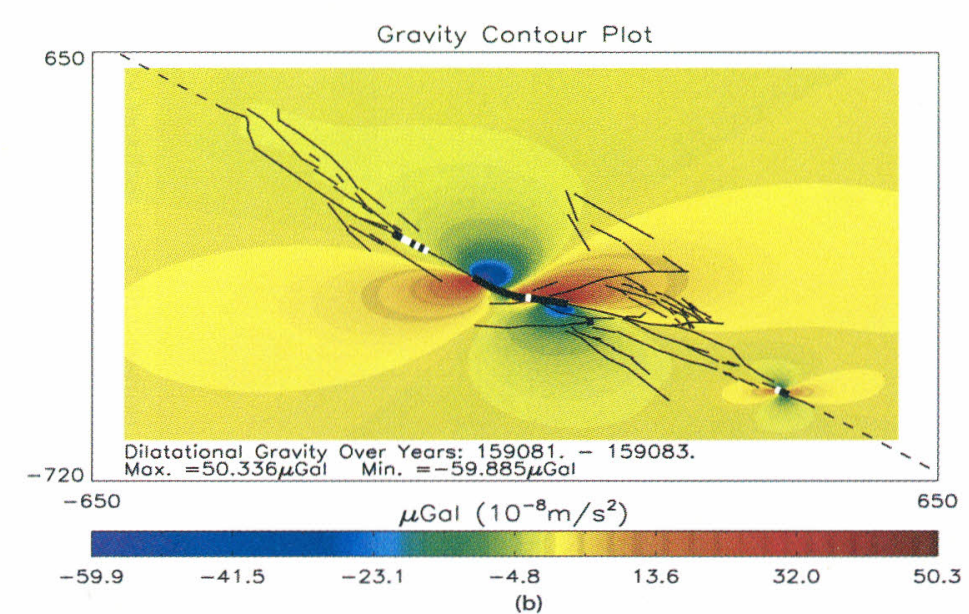

Figure 7. Gravity plots of a large event similar to the Fort Tejon earthquake of 1857. (a) Total elastic slip for each segment that slipped in the simulation. (b) Dilatational gravity signal. The key is the same as in Figure 6. 


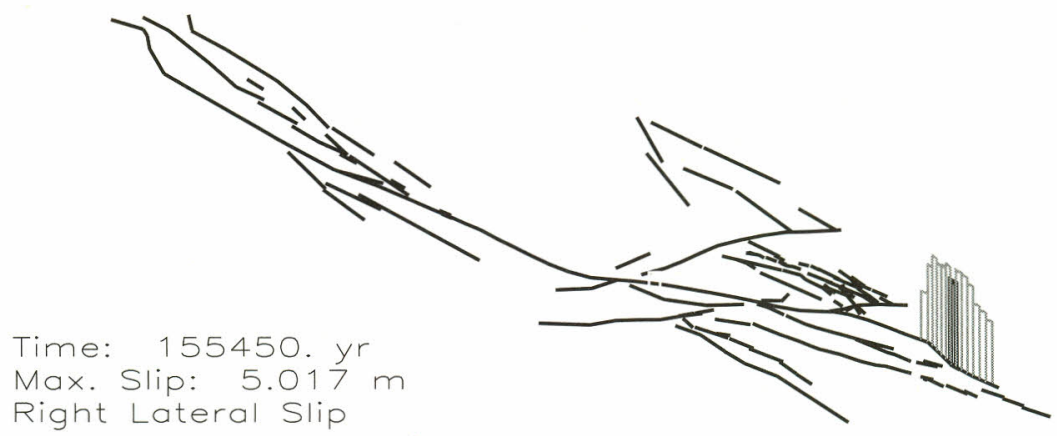

Figure 8. Modeled slip for an event at Brawley.

segments that slip with significant magnitude that are not a part of the main event. This allows us to examine the effect of the contribution almost exclusively from the complex geometry. Here we see how the "Big Bend" of the San Andreas distorts the symmetry of the signal in interesting ways. The large magnitude signals, both positive and negative, lie on the eastern portion of the fault. Conversely, on the western side of the bend, we observe a relatively smaller gravity signal, both positive and negative. Here, the stronger signal, 50.34 to $-59.85 \mu \mathrm{Gal}$, reflects the larger modeled slip magnitudes compared to the 1906 San Francisco type event segment slips. The total gravity values (not shown) range from 84.17 to $-64.68 \mu \mathrm{Gal}$. Another significant result was the location of the initiator segment (i.e., the vertical black bar in Figure 7a) for the Tejon sequence. The initiator segment is located at the Big Bend of the San Andreas fault; the same location shows an anomalous high in the dilatational steady state gravity signal in Figure 4. The dilatational high in the steady state signal reflects the locally increased density of the subsurface as a consequence of a compressional regime under continual loading.

\subsection{Gravity Evolution of a Moderate-Sized Event}

[46] We next examined a smaller event, similar to Brawley 1940 , generated by the model. Here we examine the 15 years of the accumulated gravity signal prior to the Brawley event and the event itself. This allows us to observe the evolution of the gravity signal in the region in 5 year time steps and identify regions of anomalous stress loading implied by the gravity changes.

[47] Figure 8 shows the slip modeled for the event which occurred in the model year 155450. Figures 9 to 12 show the gravity signal which develops over time starting in year 155435 , and ending with the rupture of the Brawley seg-

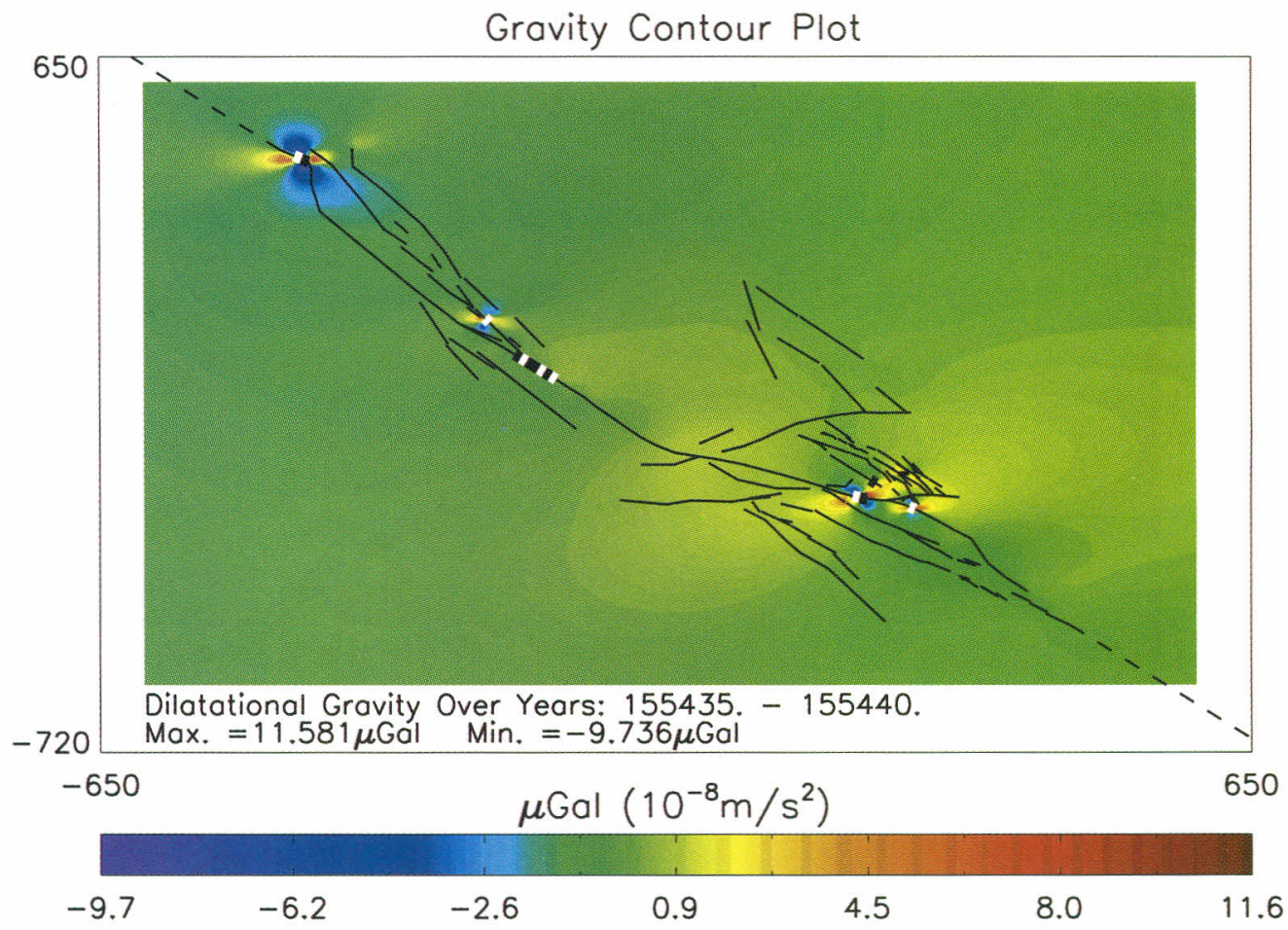

Figure 9. Five years of the accumulated dilatational gravity for Brawley ending 10 years prior to the event. 


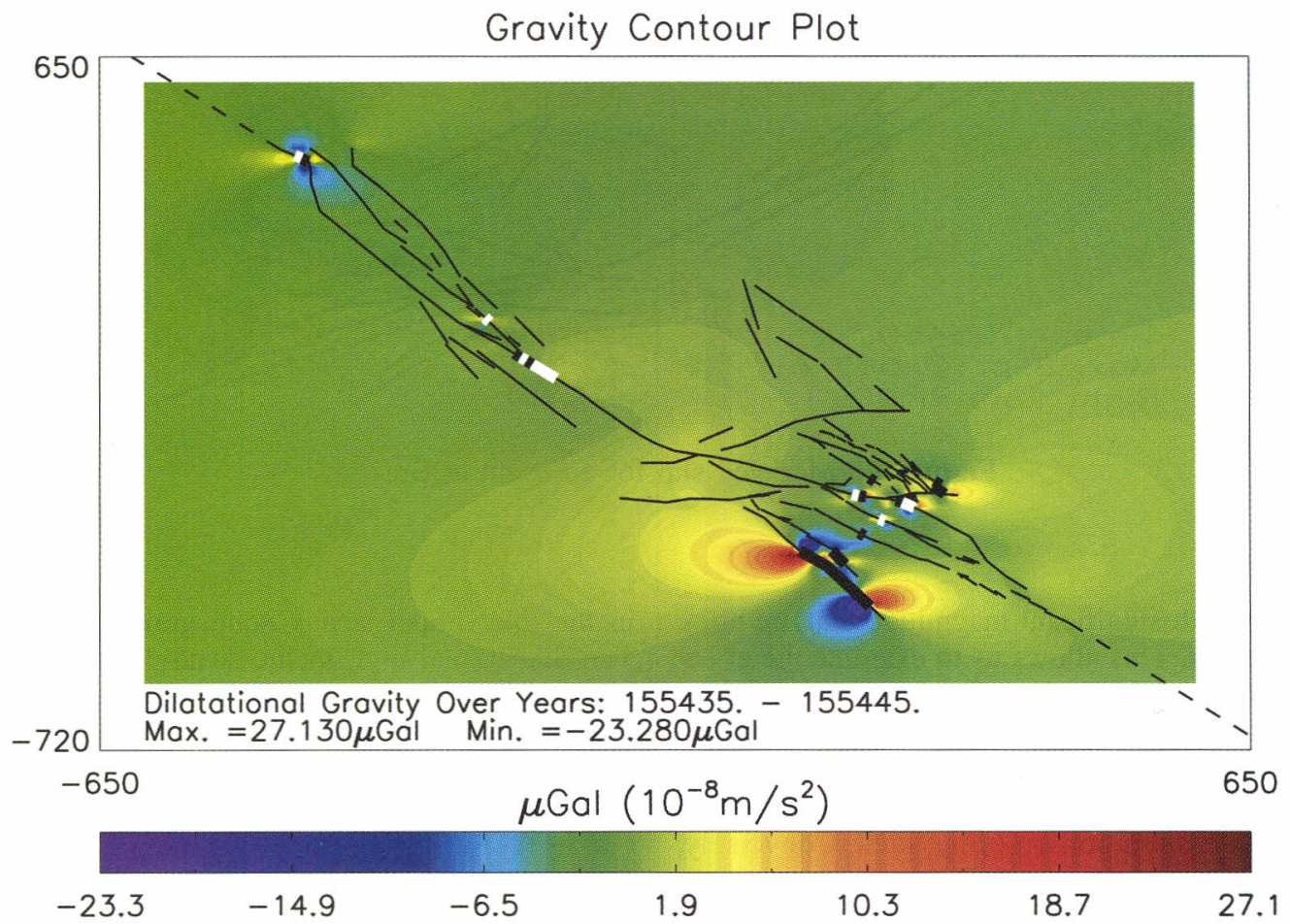

Figure 10. Ten years of the accumulated dilatational gravity for Brawley ending 5 years prior to the event.

ments in year 155450. Early on in the sequence (Figure 9), the signal is dominated by the steady state signal for the SAF system with components from small magnitude elastic slip events.
[48] In Figure 10, we see a small dilatational signal increase, $\sim 2 \mu \mathrm{Gal}$, for the Imperial Valley/Salton Sea region as well as the Santa Monica and Santa Cruz Island splay resulting from the rupture of the Coronado Bank fault

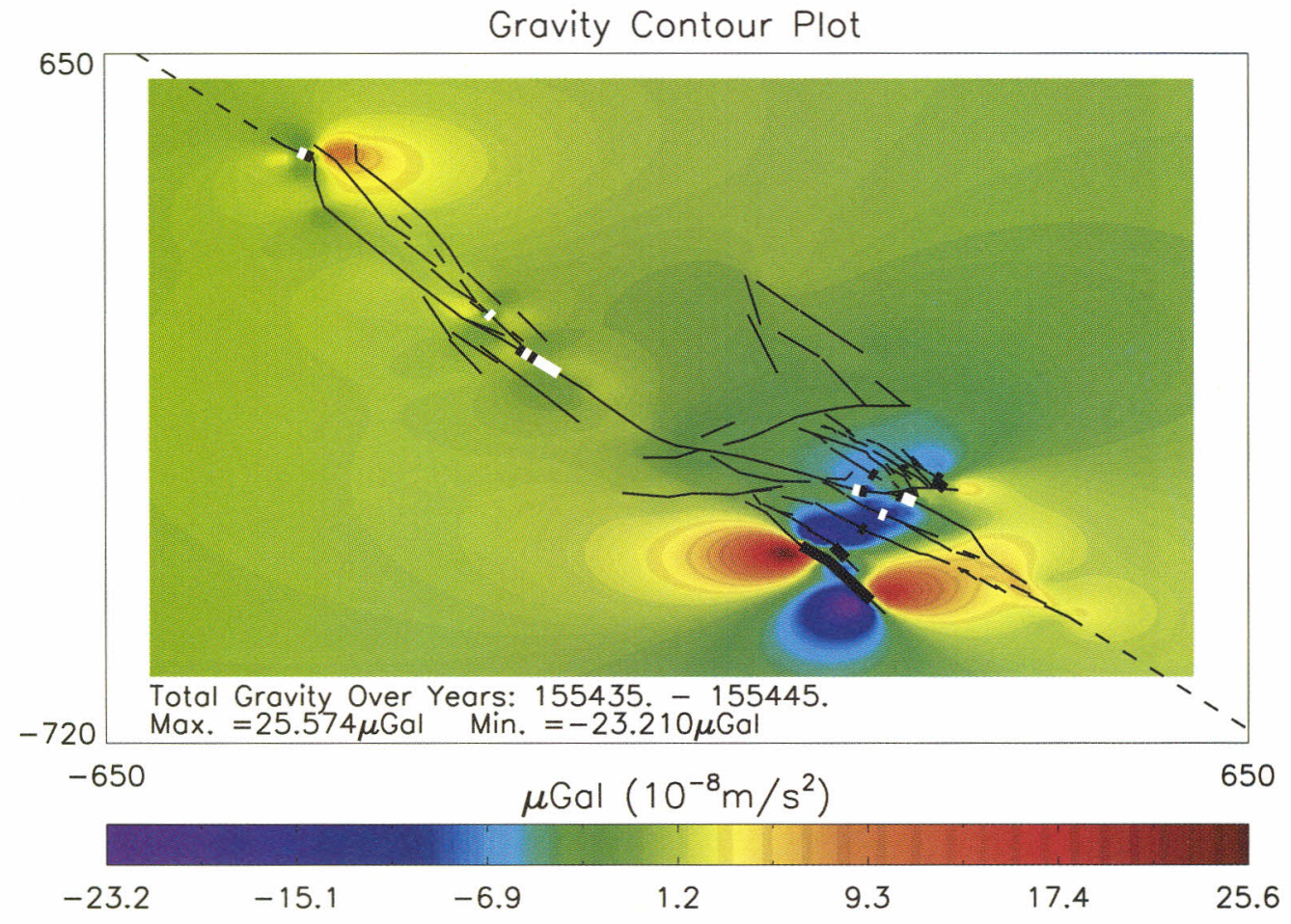

Figure 11. Ten years of the accumulated total gravity for Brawley ending 5 years prior to the event (compare Figure 10). 


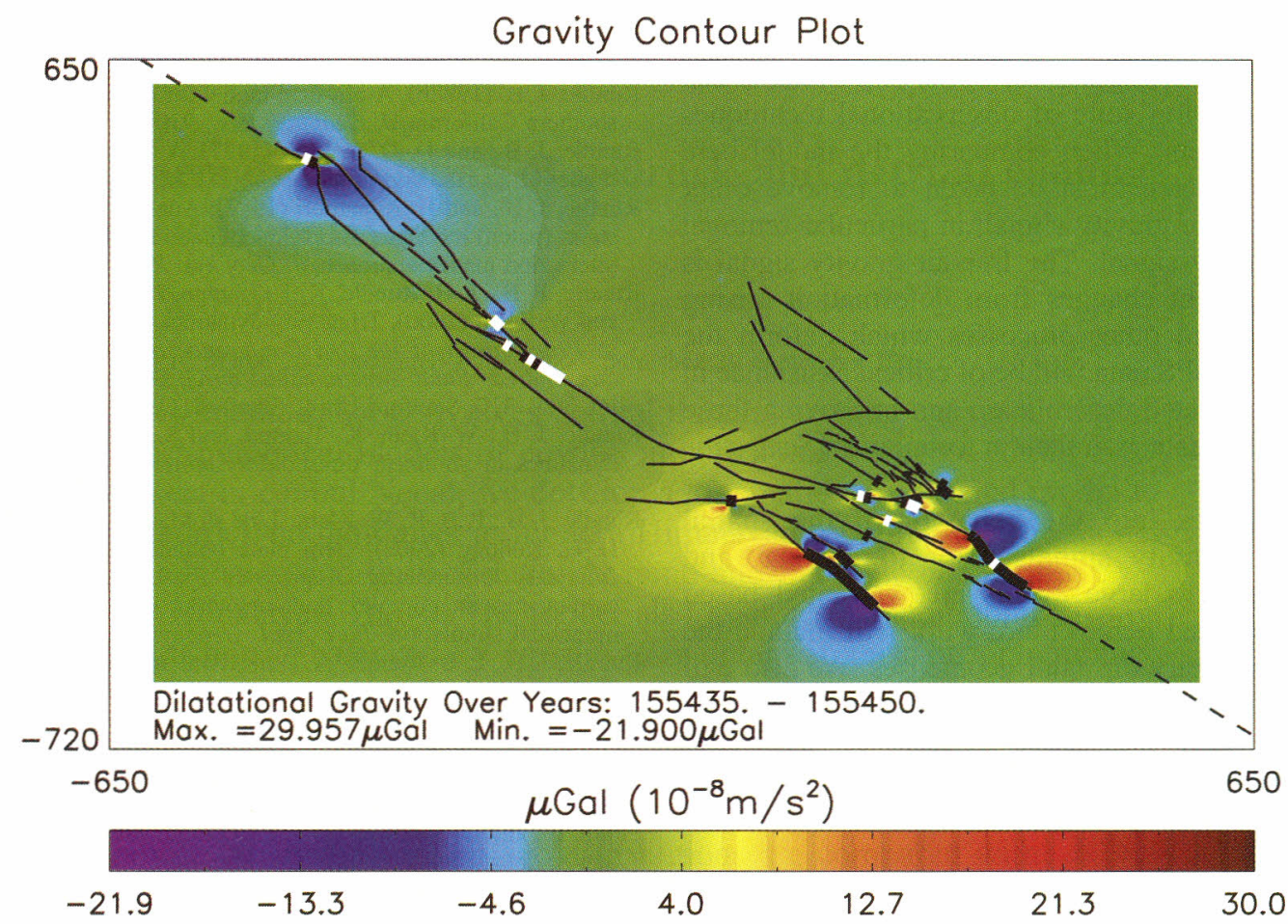

Figure 12. Fifteen years of the accumulated dilatational gravity for Brawley ending with the rupture in 155450 .

segments. Vertical deformation plays a significant role in accommodating the increased stress as demonstrated by the total gravity signal. Figure 11 shows the total gravity signal and maps out how the stress is accommodated in the region by both the subsurface dilatational regime as well as vertical deformation. Clearly, Figure 11, shows that the Imperial Valley/Salton Sea region containing the Brawley fault segments has undergone the most loading from the Coronado Bank sequence. The signal at the northern end of the San Andreas fault is due to the steady state loading of the system. As the simulation evolves, the previous year's seismic activity culminates in the model year 155450 with a rupture in the south east end of the SAF system similar to the Brawley 1940 event (Figure 12).

\section{Conclusions}

[49] The calculation of the spatial evolution of the gravity signal over a large fault network with complex geometry may act as a proxy for the strain accumulation at depth. Moreover, as gravity is an observable variable in the field, it is a likely candidate for field campaigns or permanent station deployment using traditional instruments. Here we developed a method for calculating the gravity changes over the fault network and event recurrence scales for California.

[50] Using the virtual California time-dependent stress evolution earthquake simulation to model slip histories for California, we calculated the gravity evolution over a geometrically complex fault network using the gravity Green's function solutions of Okubo [1991, 1992]. The method is general enough that the inclusion of dip-slip, tensile motion, and cavity filling matter contributions to gravity can be easily made.
[51] We found that the steady state component of the gravity signal is within the observable range of current portable instruments when taken over a 5 year period. More precise instruments, such as a superconducting gravimeter should be able to readily detect the annual component of the steady state signal. When moderate sized events occur, the gravity signal generated over large spatial areas is within field instrumentation capabilities, and well within permanent station devices such as SGs or absolute gravimeters.

[52] The presented work demonstrates that the temporal evolution of the gravity signal for strike-slip systems is also a viable candidate for field observations. Ideally, the collection of gravity data would complement the extensive preexisting GPS and seismic network in California. Permanent gravimeter sites could occupy regions of seismic interest that display persistent, measurable gravity signals in realistic fault models. Anomalous gravity signals can help identify regions of potential seismic risk and aid researchers narrow the focus of their observational efforts.

[53] Furthermore, the results of this study suggest that the SAF system is a likely candidate for periodic field campaigns of gravity data collection to specifically obtain accurate preseismic and postseismic gravity maps. Such data can potentially be assimilated into the model to further constrain the frictional parameters and, in turn, the failure recurrence intervals for each fault segment. Moreover, data collected from a large-scale field campaign can be inverse modeled to generate a more realistic picture of the current state of the subsurface stress field and can help provide forecasters with an accurate depiction of the evolution of subsurface stress; highlighting regions with anomalous signals and potentially increased future seismic risk. 
[54] We stress that the method presented here is meant to demonstrate that gravity signals for strike-slip systems can complement the existing suite of observational techniques available to researchers. When comparing the model here with collected data, care must be taken to remove other sources of noise in the gravity signal, in particular removal of the free-air gravity signal. The free-air gravity signal is dependent upon height changes from deformation arising from groundwater and other processes which deform the surface. InSAR and GPS data will be of critical assistance in confirming the associated deformation and improving these models via advanced data assimilation techniques [Samsonov and Tiampo, 2006].

[55] Further studies into the behavior of the spatial evolution of the gravity signals prior to, during, and following, moderate to large events are warranted based on the results presented here. Future work will include the incorporation of arbitrary dip fault geometries. We also will investigate the possibility of improving the method to include a viscoelastic half-space beneath an overlying elastic half-space (based on the work by Soldati et al. [1998]) to more accurately model the free-air signal in the postseismic regime.

[56] Acknowledgments. The work of T.J.H. was funded in part by the Ontario Graduate Scholarship program and the work by KFT was funded by an NSERC Discovery Grant and the Canadian Foundation for Innovation (CFI). The work of J.B.R. has been supported by a grant from U.S. Department of Energy, Office of Basic Energy Sciences, to the University of California, Davis DE-FG03-95ER14499, with additional funding from the National Aeronautics and Space Administration under grants to the University of California, Davis. Research by J.F. was funded by MEC project CGL2005-05500-C02. We wish to thank S. Okubo for his assistance as well as an anonymous reviewer and Michel Diament for their helpful criticisms and various other suggestions to improve this work.

\section{References}

Brown, R. D., Jr. (1990), Quaternary deformation, in The San Andreas fault system, California, chapter 4, pp. 83-113, U.S. Govt. Print. Off., Washington, D. C.

Chinnery, M. A. (1961), The deformation of the ground around surface faults, Bull. Seismol. Soc. Am., 51(3), 355-372.

Chinnery, M. A. (1963), The stress changes that accompany strike-slip faulting, Bull. Seismol. Soc. Am., 53(5), 921-932.

Deng, J., and L. R. Sykes (1997), Evolution of the stress field in southern California and triggering of moderate-size earthquakes: A 200 hundred year perspective, J. Geophys. Res., 102(B5), 9859-9886.

King, G. C. P., R. S. Stein, and J. Lin (1994), Static stress changes and the triggering of earthquakes, Bull. Seismol. Soc. Am. 84(3), 935-953.

Mansinha, L., and D. E. Smylie (1971), The displacement fields of inclined faults, Bull. Seismol. Soc. Am., 61(5), 1433-1440.

Maruyama, T. (1964), Statical elastic dislocations in an infinite and semiinfinite medium, Bull. Earthquake Res. Inst. Univ. Tokyo, 42, 289-368.

Massonnet, D., M. Rossi, C. Carmona, F. Adragna, G. Peltzer, K. Feigl, and T. Rabaute (1993), The displacement field of the Landers earthquake mapped by radar interferometry, Nature, 364(6433), 138-142, doi: $10.1038 / 364138 \mathrm{a} 0$

Okada, Y. (1985), Surface deformation due to shear and tensile faults in a half-space, Bull. Seismol. Soc. Am., 75(4), 1135-1154.

Okada, Y. (1992), Internal deformation due to shear and tensile faults in a half-space, Bull. Seismol. Soc. Am., 82(2), 1018-1040.

Okubo, S. (1991), Potential and gravity changes raised by point dislocations, Geophys J. Int., 105, 573-586.

Okubo, S. (1992), Gravity and potential changes due to shear and tensile faults in a half space, $J$. Geophys. Res., 97(B5), 7137-7144.

Press, F. (1965), Displacements, strains, and tilts at teleseismic distances, J. Geophys. Res., 70(10), 2395-2412.
Rundle, J. B. (1988a), A physical model for earthquakes: 1. Fluctuations and interactions, J. Geophys. Res., 93(B6), 6237-6254.

Rundle, J. B. (1988b), A physical model for earthquakes: 2. Application to southern California, J. Geophys. Res., 93(B6), 6255-6274.

Rundle, J. B., and D. D. Jackson (1977), A three-dimensional visocelastic model of a strike slip fault, Geophys. J. R. Astron. Soc., 49, 575-591.

Rundle, J. B., and H. Kanamori (1987), Application of an inhomogeneous stress (patch) model to complex subduction zone earthquakes: A discrete interaction matrix approach, J. Geophys. Res., 92(B3), 2606-2616.

Rundle, P. B., J. B. Rundle, K. F. Tiampo, J. S. S. Martins, S. McGinnis, and W. Klein (2000), Triggering dynamics on earthquake fault networks, in Proceedings of the 3rd Conference on Tectonic Problems of the San Andreas Fault System, edited by G. Bokelmann and R. L. Kovach, pp. 305-317, Stanford Univ., Stanford, Calif.

Rundle, J. B., W. Klein, K. Tiampo, and S. Gross (2001), Linear pattern dynamics in nonlinear threshold systems, Phys. Rev. E, 61(3), 24182431.

Rundle, J. B., P. B. R. W. Klein, J. de sa Martins, K. T. A. Donnellan, and L. H. Kellogg (2002), Gem plate boundary simulations for the Plate Boundary Observatory: A program for understanding the physics of earthquakes on complex fault networks via observations, theory, and numerical simulation, Pure Appl. Geophys., 159, 2357-2381.

Rundle, J. B., P. B. Rundle, A. Donnellan, and G. Fox (2004), GutenbergRichter statistics in topologically realistic system-level earthquake stressevolution simulations, Earth Planets Space, 56, 761-771.

Samsonov, S., and K. Tiampo (2006), Analytical optimization of a DInSAR and GPS dataset for derivation of three-dimensional surface motion, IEEE Geosci. Remote Sens. Lett., 3(1), 107-111.

Smith, B., and D. Sandwell (2003), Coulomb stress accumulation along the San Andreas Fault system, J. Geophys. Res., 108(B6), 2296, doi:10.1029/ 2002JB002136.

Smith, B., and D. Sandwell (2004), A three-dimensional semianalytic viscoelastic model for time-dependent analyses of the earthquake cycle, J. Geophys. Res., 109, B12401, doi:10.1029/2004JB003185.

Smith, B. R., and D. T. Sandwell (2006), A model of the earthquake cycle along the San Andreas Fault System for the past 1000 years, J. Geophys. Res., 111, B01405, doi:10.1029/2005JB003703.

Soldati, G., A. Piersanti, and E. Boschi (1998), Global postseismic gravity changes of a viscoelastic Earth, J. Geophys. Res., 103(B12), 29,86729,885 .

Song, T.-R. A., and M. Simons (2003), Large trench-parallel gravity variations predict seismogenic behavior in subduction zones, Science, 301 , $630-633$.

Stein, R. S., G. C. P. King, and J. Lin (1994), Stress triggering of the 1994 $m=6.7$ Northridge, california, earthquake by its predecessors, Science, $265,1432-1435$

Steketee, J. A. (1958a), On Volterra's dislocations in a semi-infinite elastic medium, Can. J. Phys., 36, 192-205.

Steketee, J. A. (1958b), Some geophysical applications of the elasticity theory of dislocations, Can. J. Phys., 36, 1168-1198.

Sun, W., and S. Okubo (2004), Coseismic deformations detectable by satellite gravity missions: A case study of Alaska $(1964,2002)$ and Hokkaido (2003) earthquakes in the spectral domain, J. Geophys. Res., 109, B04405, doi:10.1029/2003JB002554.

Tiampo, K. F., J. B. Rundle, S. A. McGinnis, and W. Klein (2002), Pattern dynamics and forecast methods in seismically active regions, Pure Appl. Geophys, 159(10), 2429-2467.

Tullis, T. E. (1996), Rock friction and its implications for earthquake prediction examined via models of Parkfield earthquakes, Proc. Natl. Acad Sci. USA, 93, 3803-3810.

Ward, S. N. (2000), San Francisco Bay Area earthquake simulations: A step toward a standard physical model, Bull. Seismol. Soc. Am., 90(2), 370386.

Weisbuch, G. (1991), Complex Systems Dynamics: An introduction to automata networks, Santa Fe Inst. Stud. Sci. Complex., vol. 2 , Addison-Wesley, Boston, Mass.

J. Fernández, Institutio de Astronomía y Geodesia (CSIC-UCM), Facultad de CC. Matemáticas, Ciudad Universitaria, Plaza De Ciencias 3 Madrid, 28040, Spain.

T. J. Hayes and K. F. Tiampo, Department of Earth Sciences, University of Western Ontario, 1151 Richmond St., Biological and Geological Sciences Building 158, London, ONT, Canada N6A 5B7. (thayes@uwo.ca) J. B. Rundle, Center for Computational Science and Engineering, University of California, Davis, CA 95616-0000, USA 